\title{
FUNCTIONS WHOSE FOURIER SERIES CONVERGE FOR EVERY CHANGE OF VARIABLE
}

\author{
CASPER GOFFMAN ${ }^{1}$ AND DANIEL WATERMAN ${ }^{2}$
}

1. A theorem of Pál and Bohr, [1], [2], asserts that for every continuous $f$ of period $2 \pi$ there is a homeomorphism $g$ of $[-\pi, \pi]$ with itself such that the Fourier series of $f \circ g$ converges uniformly. Salem [3] has given a powerful test for the uniform convergence of a Fourier series. On the other hand, there is no criterion which gives necessary and sufficient conditions for the Fourier series of a continuous function to converge everywhere.

In this note we show that the method of Salem may be used to determine a necessary and sufficient condition that a continuous function $f$ be such that the Fourier series of $f \circ g$ should converge everywhere for every homeomorphism $g$ of $[-\pi, \pi]$ with itself.

It is clear that this condition must be weaker than bounded variation since the continuous functions of bounded $\Phi$-variation with $\Phi=t^{p}, p>1$, have uniformly convergent Fourier series, and this class of functions is preserved by composition with homeomorphisms [3], [4].

2. We define right and left systems of intervals (at a point). Let $\left\{k_{n}\right\}$ be a sequence of positive integers such that $\lim _{n} k_{n}=\infty$ and $\lim _{n} k_{n} / n=0$.

For each $n$, let $I_{n m}, m=1, \cdots, k_{n}$, be disjoint closed intervals such that for each $n, I_{n, m-1}$ is to the left of $I_{n m}$. Let there be a real $x$ such that for every $\epsilon>0$ there is an $N$ such that $I_{n m} \subset(x, x+\epsilon)$ whenever $n>N$. Then the collection $g=\left\{I_{n m}: n=1,2, \cdots ; m=1,2, \cdots, k_{n}\right\}$ is called a right system of intervals (at $x$ ). A left system is defined similarly.

3. Let $f$ be a continuous function of period $2 \pi$ and $x$ be a point. It is easily seen that there is a sequence $\delta_{n} \searrow 0$ such that $\lim _{n} n \delta_{n}=\infty$ and

$$
\lim _{n} \int_{\delta_{n}}^{\pi} f(x \pm t) D_{n}(t) d t=0 .
$$

We need only note that if $\Delta_{k} \searrow 0$ then there exists $N_{k}$ such that

Received by the editors October 27, 1966.

1 Supported by National Science Foundation Grant No. GP 03515.

2 Supported by National Science Foundation Grant No. GP 3987. 


$$
\left|\int_{\Delta_{k}}^{\pi} f(x \pm t) D_{n}(t) d t\right|<\Delta_{k}
$$

whenever $n>N_{k}$. If $m_{k}$ is an increasing sequence of integers with $m_{k}>N_{k}$, we may set $\delta_{n}=\Delta_{k}$ for $n \in\left(m_{k}, m_{k+1}\right]$. We may choose $m_{k}$ increasing so rapidly that

$$
\lim _{n} n \delta_{n} \geqq \lim _{\boldsymbol{k}} \Delta_{k} m_{k}=\infty .
$$

It is trivial that $\lim _{n} n \delta_{n}=\infty$ implies $\lim _{n} \int_{\delta_{n}}^{\pi} D_{n}(t) d t=0$.

We have, consequently, that for $\phi(t)=f(x+t)-f(x)$ and $\psi(t)$ $=f(x-t)-f(x)$, there is a sequence $\delta_{n} \searrow 0$ with $\lim _{n} n \delta_{n}=\infty$ such that

$$
\begin{aligned}
& \lim _{n} \int_{\delta_{n}}^{\pi} \phi(t) \frac{\sin n t}{t} d t=0, \\
& \lim _{n} \int_{\delta_{n}}^{\pi} \psi(t) \frac{\sin n t}{t} d t=0 .
\end{aligned}
$$

4. We introduce the symbol $\sum(f, k, n, \theta)$ to denote

$$
\sum_{i=1}^{k} \frac{1}{i}[f((2 i) \pi / n+\theta)-f((2 i-1) \pi / n+\theta)]
$$

By combining an argument similar to that of Salem with the information available in the previous section, we obtain the following result:

For every $f$ and $x$, there is a sequence $\left\{\theta_{n}\right\}$ with $0<\theta_{n}<\pi / n$ and $a$ sequence of integers $\left\{k_{n}\right\}$ with $\lim _{n} k_{n}=\infty$ and $\lim k_{n} / n=0$ such that

$$
\int_{0}^{\pi} \phi(t) \frac{\sin n t}{t} d t
$$

and

$$
(1 / \pi) \sum\left(f, k_{n}, n, x+\theta_{n}\right)
$$

are equiconvergent.

A similar result holds for the left side of $x$.

A suitable choice of $k_{n}$ would be, for example, $\left[n \delta_{n} / 2 \pi-1 / 2\right]$.

5. This result yields a criterion for everywhere convergence of Fourier series which is invariant under changes in variable.

For every right system, $\mathfrak{g}$, consider the sequence

$$
\alpha_{n}(\mathfrak{g})=\sum_{i=1}^{k_{n}} \frac{1}{i} f\left(I_{n i}\right)
$$


where, for any interval $I=[a, b], f(I)=f(b)-f(a) . \alpha_{n}(g)$ is defined similarly for left systems.

Suppose now that $f$ is such that $\alpha_{n}(\mathfrak{g})$ converges to zero for every right and left system. Then, for every $x$, we may shrink the intervals $\left[x+\theta_{n}+(2 i-1) \pi / n, x+\theta_{n}+2 i \pi / n\right], i=1, \cdots, k_{n}$, to obtain disjoint closed intervals $I_{n i}, i=1, \cdots, k_{n}$, so that $\alpha_{n}(g)$ is equiconvergent with $\sum\left(f, k_{n}, n, x+\theta_{n}\right)$. This yields the following result:

If $f$ is such that $\alpha_{n}(g)$ converges to zero for every right and left system $g$ then, for every homeomorphism $g$, the Fourier series of $f \circ g$ converges everywhere.

6. We now show that if $f$ is such that there is a right (or left) system at a point $x$ for which $\lim _{n} \alpha_{n}(g) \neq 0$, then there is a homeomorphism $g$ such that the Fourier series of $f \circ g$ diverges at $x$. We assume, as we may, that $g$ is a right system, $x=0, f(0)=0$ and for some finite $\alpha>0, \lim _{n} \sup \alpha_{n}(\mathfrak{g}) \geqq \alpha$.

We first describe briefly the essential idea behind the construction. Let $I_{n i}=\left[\tau_{n i}, \tau_{n i}^{\prime}\right]$ for $I_{n i} \in \mathfrak{g}$. We choose an increasing sequence of integers $\left\{m_{n}\right\}$ such that $\left(2 k_{m_{n+1}}+1\right) / m_{n+1}<1 / m_{n}, \tau_{m_{n+1}}^{\prime} k_{m_{n+1}}<\tau_{m_{n} 1}$, and $\alpha_{m_{n}}>\alpha\left(1-1 / 2^{n}\right)$. If $g$ is constant on each interval $\left(i \pi / m_{n}\right.$, $\left.(i+1) \pi / m_{n}\right), i=1, \cdots, 2 k_{m_{n}}$, assuming the values $\tau_{m_{n} 1}, \tau_{m_{n} 1}^{\prime}, \tau_{m_{n} 2}$, $\tau_{m_{n} 2}^{\prime}, \cdots$ successively, then, for $\left\{m_{n}\right\}$ increasing sufficiently rapidly, we can define $g$ as a piecewise constant, nondecreasing function on $[0, \pi]$ so that

$$
\int_{0}^{\left(2 k_{m_{n}}+1\right) \pi / m_{n}}(f \circ g)(t) \frac{\sin m_{n} t}{t} d t
$$

and $\alpha_{m_{n}} / \pi$ are equiconvergent and

$$
\lim _{n} \int_{\left(2 k_{m_{n}}+1\right) \pi / m_{n}}^{\pi}(f \circ g)(t) \frac{\sin m_{n} t}{t} d t=0 .
$$

The $g$ thus defined can be altered to give a piecewise linear, continuous, increasing function which has small slope on most of each of the former intervals of constancy and rises abruptly on a small portion of each of these intervals. This can be done so as to preserve the properties of $g$ noted above.

It is somewhat simpler, however, to construct the homeomorphism $g$ directly. This is accomplished by the use of the following

LeмmA. Let $\left\{k_{n}\right\}$ be a sequence of integers with the properties $\lim _{n} k_{n}=\infty, \lim _{n} k_{n} / n=0$. There is a sequence $\left\{\epsilon_{n}\right\}, 0<\epsilon_{n}<\pi / n$, such that for every function $h$ continuous in a neighborhood of zero with 
$h(0)=0$ there is a sequence $\left\{\theta_{n}\right\}, 0<\theta_{n}<\pi / n-\epsilon_{n}$, such that

$$
\int_{0}^{\left(2 k_{n}+1\right) \pi / n} h(t) \frac{\sin n t}{t} d t
$$

and $(1 / \pi) \sum\left(h, k_{n}, n, \theta_{n}\right)$ are equiconvergent.

Proof. We write

$$
\int_{0}^{\left(2 k_{n}+1\right) \pi / n} h(t) \frac{\sin n t}{t} d t=\int_{0}^{\pi / n} \cdots+\sum_{i=1}^{2 k_{n}} \int_{i \pi / n}^{(i+1) \pi / n} \cdots=P+Q .
$$

We have

$$
|P| \leqq C \sup _{t \in(0, \pi / n)}|h(t)|=o(1)
$$

as $n \rightarrow \infty$ and, for small $\eta_{n} \in(0, \pi)$,

$$
\begin{aligned}
Q & =\int_{0}^{\pi} \sin t \sum_{i=1}^{2 k_{n}}(-1)^{i} \frac{h((t+i \pi) / n)}{t+i \pi} d t \\
& =\int_{0}^{\pi-\eta_{n}} \cdots+\int_{\pi-\eta_{n}}^{\pi} \cdots \\
& =\left(1+\cos \eta_{n}\right) \sum_{i=1}^{2 k_{n}}(-1)^{i} \frac{h\left(\theta_{n}+i \pi / n\right)}{n \theta_{n}+i \pi}+R
\end{aligned}
$$

where $\theta_{n} \in\left(0,\left(\pi-\eta_{n}\right) / n\right)$. Now

$$
|R| \leqq \eta_{n}^{2} \cdot \frac{1}{\pi} \sum_{i=1}^{2 k_{n}} \frac{1}{i} \cdot \sup _{t \in\left(0,\left(2 k_{n}+1\right) \pi / n\right)}|h(t)|=o(1)
$$

as $n \rightarrow \infty$ if $\left.\eta_{n}=O\left(1 / \log k_{n}\right)^{1 / 2}\right)$ since $k_{n} / n \rightarrow 0$ as $n \rightarrow \infty$.

We note now that

$$
\begin{aligned}
\frac{h\left(\theta_{n}+2 i \pi / n\right)}{n \theta_{n}+2 i \pi}- & \frac{h\left(\theta_{n}+(2 i-1) \pi / n\right)}{n \theta_{n}+(2 i-1) \pi} \\
= & \left(h\left(\theta_{n}+2 i \pi / n\right)-h\left(\theta_{n}+(2 i-1) \pi / n\right)\right) /\left(n \theta_{n}+2 i \pi\right) \\
& -h\left(\theta_{n}+(2 i-1) \pi / n\right) \pi /\left(n \theta_{n}+(2 i-1) \pi\right)\left(n \theta_{n}+2 i \pi\right) .
\end{aligned}
$$

If we drop the sum of the last terms, the error is less than

$$
C \sup _{t \in\left(0,\left(2 k_{n}+1\right) \pi / n\right)}|h(t)|=o(1)
$$

as $n \rightarrow \infty$. Next we have

$$
\left|1 /\left(n \theta_{n}+2 i \pi\right)-1 / 2 i \pi\right|<1 / 4 \pi i^{2}
$$


and so if we replace $n \theta_{n}+2 i \pi+2 i \pi$ by $2 i \pi$, the error is again less than

$$
C \sup _{t \in\left(0,\left(2 k_{n}+1\right) \pi / n\right)}|h(t)|=o(1)
$$

as $n \rightarrow \infty$. If we replace $\left(1+\cos \eta_{n}\right)$ by 2 we introduce an error of less than

$$
\begin{aligned}
\left(1-\cos \eta_{n}\right) \cdot \frac{1}{\pi} \mid \sum_{i=1}^{k_{n}}\left(h \left(\theta_{n}+\right.\right. & \left.2 i \pi / n)-h\left(\theta_{n}+(2 i-1) \pi / n\right)\right) / 2 i \mid \\
& <C \eta_{n}^{2} \log k_{n} \sup _{t \in\left(0,\left(2 k_{n}+1\right) \pi / n\right)}|h(t)|=o(1)
\end{aligned}
$$

as $n \rightarrow \infty$ if $\eta_{n}=O\left(1 /\left(\log k_{n}\right)^{1 / 2}\right)$.

Letting $\epsilon_{n}=\eta_{n} / n=O\left(1 / n\left(\log k_{n}\right)^{1 / 2}\right)$ the proof of the lemma is complete. We proceed with the construction of the homeomorphism $g$.

We choose an integer $m_{1}$ such that

$$
\alpha_{m_{1}}(\mathfrak{g})>\alpha / 2
$$

and so large that for some $b_{1} \in\left(\tau_{m_{1} \mathbf{k}_{m_{1}}}^{\prime}, \pi\right)$ and sufficiently close to $\pi$ we have

$$
\left|\int_{\left(2 k_{m_{1}}+1\right) \pi / m_{1}}^{\pi}(f \circ g)(t) \frac{\sin m_{1} t}{t} d t\right|<1 / 2,
$$

where $g$ is the increasing linear function mapping $\left[\left(2 k_{m_{1}}+1\right) \pi / m_{1}, \pi\right]$ onto $\left[b_{1}, \pi\right]$. To show that such a choice of $m_{1}$ and $b_{1}$ is possible, we note that the integral in $\left(\mathrm{ii}_{1}\right)$ is less than

$$
|f(\pi)|\left|\int_{\left(2 k_{m_{1}}+1\right) \pi / m_{1}}^{\pi} \frac{\sin m_{1} t}{t} d t\right|+\sup _{t \in\left(b_{1}, \pi\right)}|f(\pi)-f(t)| C \log m_{1} .
$$

The first term can be made small by choosing $m_{1}$ large and, for any choice of $m_{1}$, the second term will be small if $b_{1}$ is sufficiently close to $\pi$.

We now set

$$
g\left((2 i-1) \pi / m_{i}\right)=\tau_{m_{1}}, \quad g\left(2 i \pi / m_{1}\right)=\tau_{m_{1} i}^{\prime}, \quad i=1, \cdots, k_{m_{1}},
$$

and let $g$ be linear on each interval $\left[i \pi / m_{1},(i+1) \pi / m_{1}-\epsilon_{m_{1}}\right]$, $i=1, \cdots, 2 k_{m_{1}}$, and on each interval $\left[i \pi / m_{1}-\epsilon_{m_{1}}, i \pi / m_{1}\right], i=1, \cdots$, $2 k_{m_{1}}+1$, and be continuous and increasing on $\left[\pi / m_{1}, \pi\right]$. The slope of $g$ on each interval $\left[i \pi / m_{1},(i+1) \pi / m_{1}-\epsilon_{m_{1}}\right]$ is chosen so small that (iii $\left.{ }_{1}\right)$

$$
\left|\alpha_{m_{1}}(g)-\sum\left(f \circ g, k_{m_{1}}, m_{1}, \theta\right)\right|<1 / 2
$$

for every $\theta \in\left(0, \pi / m_{1}-\epsilon_{m_{1}}\right)$. 
Suppose now that we have defined $m_{1}<\cdots<m_{r}$ and a homeomorphism $g$ from $\left[\pi / m_{r}, \pi\right]$ onto $\left[\tau_{m_{r} 1}, \pi\right], g(\pi)=\pi$, such that for $n=1, \cdots, r$ we have

$$
\begin{gathered}
\alpha_{m_{n}}(g)>\alpha\left(1-1 / 2^{n}\right), \\
\left|\int_{\left(2 k_{m_{n}}+1\right) \pi / m_{n}}^{\pi}(f \circ g)(t) \frac{\sin m_{n} t}{t} d t\right|<1 / 2^{n},
\end{gathered}
$$

and

$\left(\mathrm{iii}_{n}\right)\left|\alpha_{m_{n}}(g)-\sum\left(f \circ g, k_{m_{n}}, m_{n}, \theta\right)\right|<1 / 2^{n}, \quad \forall \theta \in\left(0, \pi / m_{n}-\epsilon_{m_{n}}\right)$.

We now choose $m_{r+1}$ such that $\left(2 k_{m_{r+1}}+1\right) / m_{r+1}<1 / m_{r}, \tau_{m_{r+1}, k_{n_{r+1}}}^{\prime}$ $<\tau_{m_{r} 1}$, and $\left(\mathrm{i}_{r+1}\right)$ is satisfied.

This $m_{r+1}$ may be chosen so large that $\left(\mathrm{ii}_{r+1}\right)$ is satisfied when $g$ is extended as the increasing linear mapping of $\left[\left(2 k_{m_{r+1}}+1\right) \pi / m_{r+1}, \pi / m_{r}\right]$ onto $\left[b_{r+1}, \tau_{m_{r} 1}\right]$, where $b_{r+1} \in\left(\tau_{m_{r+1}, k_{m_{r+1}}}^{\prime}, \tau_{m_{r} 1}\right)$ and is sufficiently close to $\tau_{m_{r}}$.

We now extend $g$ as an increasing, continuous, piecewise linear function from $\left[\pi / m_{r+1},\left(2 k_{m_{r+1}}+1\right) \pi / m_{r+1}\right]$ onto $\left[\tau_{m_{r+1} 1}, b_{r+1}\right]$ in a manner analogous to the definition of $g$ on $\left[\pi / m_{1},\left(2 k_{m_{1}}+1\right) \pi / m_{1}\right]$. The slope of $g$ on each interval $\left[i \pi / m_{r+1},(i+1) \pi / m_{r+1}-\epsilon_{m_{r+1}}\right]$ is chosen so small that $\left(\mathrm{iii}_{r+1}\right)$ holds.

Thus we see that we can define an increasing sequence of integers $\left\{m_{n}\right\}$ with properties $\left(\mathrm{i}_{n}\right)$, (iii $)$, and $\left(\mathrm{iii}_{n}\right)$ for all $n$. We extend the definition of $g(t)$ to $[0, \pi]$ by setting $g(0)=0$ and we have at once that $g$ is a homeomorphism of $[0, \pi]$ with itself such that

$$
\liminf _{n} \int_{0}^{\pi}(f \circ g)(t) \frac{\sin m_{n} t}{t} d t \geqq \alpha / \pi .
$$

It is relatively easy to extend $g$ to a homeomorphism of $[-\pi, \pi]$ with itself such that

$$
\limsup _{n} \int_{-\pi}^{0}(f \circ g)(t) \frac{\sin m_{n} t}{t} d t \geqq 0 .
$$

This proves the

ThEOREM. $f$ is such that $f \circ g$ has an everywhere convergent Fourier series for every homeomorphism $g$ if and only if $\lim _{n} \alpha_{n}(\mathfrak{g})=0$ for every system $\mathrm{g}$.

It should be noted that the requirement on a system $g$ that the $I_{n m}, m=1, \cdots, k_{n}$, be disjoint is not essential and can be replaced by 
nonoverlapping, for if we assume that $\mathscr{g}$ satisfies the latter condition and $\lim \alpha_{n}(g) \neq 0$, then by shrinking the intervals of $\mathscr{g}$ slightly we may obtain an $g^{\prime}$ satisfying the former condition for which lim $\alpha_{n}\left(\mathcal{I}^{\prime}\right) \neq 0$.

\section{REFERENCES}

1. J. Pál, Sur des transformations de fonctions qui font converger leurs séries de Fourier, C.R. Acad. Sci. Paris 158 (1914), 101-103.

2. H. Bohr, Über einem Satz von J. Pal, Acta Szeged 7 (1935), 129-135.

3. R. Salem, Essais sur les séries trigonométrique, Actualities Sci. Ind. No. 862, Hermann, Paris, 1940.

4. L. C. Young, Sur une généralisation de la notion de variation de puissance $p$ ième bornée au sens de $M$. Wiener, et sur la convergence des séries de Fourier, C. R. Acad. Sci. Paris 204 (1937), 470-472.

Purdue University and

WAYNE UNIVERSITY 